\title{
Entrelacs
}

Cinéma et audiovisuel

14 | 2018

Marchés du film : évolutions, mutations et perspectives

\section{Les pratiques professionnelles au sein du marché cinématographique : La relation entre distributeurs et exploitants}

Clémence Allamand

\section{OpenEdition}

\section{Journals}

Édition électronique

URL : http://journals.openedition.org/entrelacs/4204

DOI : 10.4000 /entrelacs.4204

ISSN : 2261-5482

Éditeur

Éditions Téraèdre

Référence électronique

Clémence Allamand, «Les pratiques professionnelles au sein du marché cinématographique : La relation entre distributeurs et exploitants », Entrelacs [En ligne], 14 | 2018, mis en ligne le 17 décembre 2018, consulté le 01 mai 2019. URL : http://journals.openedition.org/entrelacs/4204 ; DOI : 10.4000/ entrelacs.4204

Ce document a été généré automatiquement le 1 mai 2019.

Tous droits réservés 


\title{
Les pratiques professionnelles au sein du marché cinématographique : La relation entre distributeurs et exploitants
}

\author{
Clémence Allamand
}

1 S'intéresser aux rapports entre le métier de distributeur et celui d'exploitant revient à s'intéresser aux relations contractuelles relatives à la programmation des films. Le distributeur loue les droits d'exploitation d'un film à une salle, c'est-à-dire qu'il détermine avec le programmateur le taux de location de la copie du film¹ ${ }^{1}$. Ces droits sont négociés soit à la semaine - du mercredi au mercredi -, soit au mois. Dans un certain nombre de cas - près de la moitié -, l'exploitant n'est pas le programmateur de la salle et c'est à un "troisième homme " qu'incombe cette tâche. Il peut s'agir d'une seule personne, en charge de la programmation d'un groupement d'exploitation, ou bien d'une société intermédiaire, une entente de programmation ${ }^{2}$, qui programme plusieurs cinémas indépendants. C'est dans le cadre de l'acte de programmation que nous intéresse la relation entre distributeurs et exploitants, puisqu'en réalité, dans le cas où l'exploitant n'est pas programmateur de sa salle, les relations avec les distributeurs sont quasi inexistantes ${ }^{3}$.

2 Bien que les négociations entre distributeurs et exploitants-programmateurs donnent lieu à un accord sur le taux de location d'un film donné et à un engagement possible sur un certain nombre de séances, parfois de semaines, il s'agit d'un contrat verbal; il n'existe aujourd'hui pratiquement plus de contrat papier signét. Si ces derniers se faisaient de plus en plus rares depuis la fin des années 1990, le passage à la projection numérique à la fin des années 2000 a fait cesser cette pratique, notamment suite à la disparition de la copie physique du film ${ }^{5}$, les programmateurs ne raisonnant plus en termes de copies, mais en nombre de salles. Les négociations s'effectuent par téléphone ou par mail et l'accord conclu repose uniquement sur la bonne foi des deux parties. 
3 Les pratiques des professionnels de l'industrie cinématographique sont peu étudiées dans les travaux académiques. Si des articles sur le métier de programmateur existent, à l'instar des travaux de Claude Forest ${ }^{6}$ ou de Sarah Budex ${ }^{7}$, cet article a pour visée, s'appuyant sur une enquête de terrain approfondie ${ }^{8}$, d'étudier spécifiquement le fonctionnement des relations entre les distributeurs et les exploitants sur le marché cinématographique par le prisme des discours portés par et sur les différents acteurs afin de mieux appréhender l'organisation du secteur et les rapports de force à l'œuvre au sein de celui-ci. Il s'agit de montrer que leur relation ne relève pas seulement d'un enjeu commercial évident lié à la programmation des films, mais d'une relation de pouvoir qui s'organise aussi autour de régimes de valeurs essentielles à la construction d'identités collectives ${ }^{9}$, dans un contexte où, pour reprendre les termes de Michel Gomez, « le poids de la loi et du réglementaire y est faible et le poids des habitudes, bonnes ou mauvaises, est important tout comme l'expression des rapports de force propre à toute filière ${ }^{10} »$. S'il y a indubitablement une divergence d'intérêts entre les distributeurs et les exploitants, c'est également le cas au sein même des deux métiers. La diversité apparente du champ économique et social des acteurs de la diffusion cinématographique engendre des rapports interprofessionnels divers, dominés selon les situations et les acteurs mis en relation par des intérêts ou des objectifs d'ordre différent. Ces logiques sociales, « autour desquelles les stratégies des acteurs sociaux quels qu'ils soient sont plus ou moins contraints de s'organiser et de se développer $"{ }^{11}$, oscillent entre plusieurs types de déterminations : choix artistiques, rentabilité économique voire profit, et rapports de force liés aux relations de pouvoir et aux interdépendances que cela engendre. Suivant le contexte, certaines déterminations prédominent et induisent largement l'issue de l'échange entre les acteurs.

\section{Des rapports de force entre des distributeurs, des exploitants autour des films}

4 Olivier Bomsel et Gilles Leblanc résument l'enjeu principal de la relation entre distributeurs et exploitants ainsi :

La clé de la relation distributeur-exploitant est dans le contrat de location du film. La question est de savoir comment se partage le risque commercial, et derrière cela, qui vend quoi et comment: l'exploitant vend-il au distributeur la clientèle de sa salle ou, au contraire, le distributeur vend-il à la salle la clientèle de son film ${ }^{12}$ ?

5 Une réponse possible serait de dire que cela dépend du film. Les distributeurs et les exploitants travaillent avec des films qu'ils estiment «forts ", d'autres « faibles », c'est-àdire qu'ils ont un fort ou un faible potentiel commercial ${ }^{13}$. Ils parlent également d' d' art et $^{2}$ essai porteur ", ce qui correspond peu ou prou à l'existence d'un ou plusieurs éléments tels la présence d'un réalisateur confirmé, un prix dans des festivals importants, un "gros» casting, un "buzz» autour du film, etc., mais qui tend à rester une sorte d'énigme contemporaine, ces films n'obtiennent pas toujours de bons résultats en termes d'entrées. Cette typologie sans fin montre bien toutes les difficultés - la part d'incertitude étant très importante - face auxquelles une économie de prototypes est confrontée.

6 La relation entre distributeurs et exploitants relève donc d'un enjeu avant tout commercial, puisque le distributeur cherche à rentabiliser ses copies, et l'exploitant ses fauteuils ${ }^{14}$. De là découle un rapport de force correspondant aux intérêts économiques 
propres qui peuvent diverger ou converger en fonction des films, et qui place, selon la situation, le distributeur ou l'exploitant en position de force. Cette relation de dominantdominé s'inverse selon les types d'acteurs et les situations de négociation. En outre, le rapport de force ne s'exerce pas toujours en faveur d'une même salle ou d'un même distributeur, mais il dépend également fortement des films distribués. C'est un rapport de force mouvant. Du côté de l'exploitation, un multiplexe possédé par un circuit d'exploitation type UGC ou Pathé-Gaumont sera habituellement dans une position de force face à n'importe quel distributeur. La salle indépendante n'a en revanche souvent pas d'autre choix que d'accepter une sortie en tandem - le même film programmé dans la même zone de chalandise - ou bien d'y renoncer complètement. Parallèlement, un distributeur indépendant peut aussi bien se retrouver dans une situation de faiblesse dans les négociations si les exploitants - gros ou petits - ne s'intéressent pas à son film, ou dominer le rapport de force si le film est très demandé.

7 Il faut de surcroît ajouter à cette relation complexe la concurrence sur le marché de la diffusion des distributeurs entre eux au sein d'un contexte ultra-concurrentiel dans lequel 12 à 15 films sortant chaque semaine - certains sur des combinaisons de sorties avoisinant les 1000 copies - doivent se partager un peu plus de 5800 écrans. Les distributeurs peuvent avoir du mal à placer leur film et à tendre vers le nombre de copies initialement souhaité lors de l'élaboration du plan de sortie si un film concurrent en termes de public(s) cible(s) est programmé la même semaine. Prenons l'exemple de la semaine du 31 janvier 2018, pendant laquelle l'exploitant aura le choix de programmer des films présentant des atouts marketing différents, notamment Gaspard va au Mariage ${ }^{15}$, film français avec Félix Moati distribué par un indépendant, et Wonder Whee ${ }^{16}$, le dernier film de Woody Allen. Ces films ont potentiellement en commun le même public. De même, pour un distributeur indépendant, sortir un film art et essai la semaine du 14 février 2018 peut s'avérer compliqué face à Phantom Thread ${ }^{17}$ et à l'Apparition $^{18}$, qui présentent de forts atouts marketing: pour le premier, le réalisateur Paul Thomas Anderson et l'acteur principal Daniel Day-Lewis (qui a de surcroît annoncé mettre fin à sa carrière d'acteur), nommé plusieurs fois aux Oscars, et pour le second, le fait qu'il s'agisse du dernier film de Xavier Giannoli avec Vincent Lindon en tête d'affiche. Cette configuration rend plus difficile la programmation d'un autre film art et essai en cette même semaine. Pour les indépendants, le nerf de la guerre concerne le fameux « art et essai porteur ", car c'est à ce niveau-là que se situe la concurrence.

8 Les rapports de force qui s'exercent entre les distributeurs et les exploitants, voire entre les acteurs au sein même de chacun des deux secteurs montrent qu'il s'agit avant tout d'une relation de pouvoir. Plus la part d'indétermination dans le résultat des choix de programmation est réduite, plus les acteurs exercent du pouvoir; pour reprendre l'expression de Michel Crozier, «le pouvoir, c'est la maîtrise de l'incertitude ${ }^{19}$ ». Ce sont donc les distributeurs qui ont « les films que toutes les salles veulent », et « les salles dans lesquelles tous les distributeurs veulent sortir leurs films » qui possèdent du pouvoir.

Comment analyser cette relation de pouvoir? Autrement dit, comment, dans le contexte décrit ci-dessus, les distributeurs et les exploitants, aussi divers soient-ils, négocient-ils la programmation des films? Nous avons pu voir que les acteurs de la diffusion poursuivent des intérêts divergents, mais ces intérêts peuvent être plus aisément satisfaits si tous concourent à la réussite d'un objectif commun, celui du succès du film. Pour cela, ils doivent donc travailler ensemble dans l'intérêt du film pour que celui-ci tende à une programmation qui soit le plus en adéquation avec son potentiel commercial - que le 
distributeur a pour mission d'estimer. C'est pourquoi il importe de s'intéresser davantage aux habitudes de travail et aux pratiques pour mieux comprendre le processus de négociation dans la programmation des films.

\section{La nécessité de se démarquer}

10 Les pratiques des distributeurs et des exploitants, qu'elles relèvent davantage de l'habitude ou d'un calcul stratégique, vont être orientées de façon plus ou moins conscientisée par la manière dont s'exerce le pouvoir. Autrement dit, ce sont les structurations de pouvoir qui définissent la rationalité des stratégies ${ }^{20}$.

Nous l'avons dit, le travail des programmateurs-distributeurs consiste à négocier avec les programmateurs de salles pour espérer s'approcher au maximum du plan de sortie élaboré, c'est-à-dire tendre vers la combinaison de copies - le nombre d'écrans - pensée idéalement. Il ne s'agit donc pas simplement de déterminer un nombre de copies et de financer ce déploiement. Programmer un film dans une salle signifie avant tout pour un distributeur avoir réussi à convaincre l'exploitant de programmer son film. Le distributeur doit donc "vendre » - au sens marketing du terme - son film à la salle. Pour cela, il est important de donner une identité au film. Le positionnement du film est défini par le distributeur et se retrouve dans les choix de promotion et de programmation $\mathrm{du}$ film. Afin de tenter de réduire les aléas inhérents à la commercialisation de tout produit culturel, le distributeur se réfère au plan de sortie de films qu'il considère comme « similaires» (souvent relatif au genre du film) afin de l'utiliser comme base de travail, tout en tenant compte des spécificités du nouveau film en particulier pour déterminer le type de salles qui sera privilégié. Il est également attentif aux spécificités régionales (davantage de copies dans la région dans laquelle le film a été tourné par exemple) ou en fonction des diffusions en festivals (établir une continuité de programmation dans une localisation où le film est connu).

Face à une offre de films très abondante où « hyperchoix » rime avec « difficulté de faire un choix ", et face aux pratiques des " gros distributeurs » qui optent régulièrement pour des stratégies d'offre saturante, les films les plus visibles deviennent facilement les plus choisis et a fortiori les fameux « films que tout le monde veut programmer/voir ». Dans ce contexte, il faut donc que les films se démarquent les uns des autres, qu'ils présentent un avantage concurrentiel que le distributeur va mettre en avant, voire créer de toutes pièces. De ce fait, selon une logique de marketing de l'offre définie par Hélène Laurichesse, le distributeur s'inscrit dans « une démarche de médiation entre l'offre - ici le distributeur - et la demande - l'exploitant qui fait des choix pour ses spectateurs afin de permettre à une œuvre achevée de rencontrer son public ${ }^{21} »$.

\section{Postures et stratégies de négociations}

13 Comment le distributeur peut-il convaincre ? Il doit montrer à l'exploitant l'intérêt que présente le film, parce que c'est un «bon film», parce qu'il possède de l'attrait commercial, parce qu'il est possible de mener une politique d'animation locale intéressante en sollicitant telle ou telle association en rapport avec la thématique du film en question, parce qu'il permet de faire découvrir un nouveau réalisateur, de nouveaux acteurs et défendre ainsi la diversité culturelle, etc. Tous les films ne présentent pas les 
mêmes atouts, et il est dans un premier temps indispensable de faire connaitre le film, en permettant notamment aux exploitants de le visionner suffisamment en amont pour qu'ils puissent l'envisager dans leur programmation. Lorsque le distributeur sollicite l'exploitant pour lui proposer son film, il le «pitche»- exercice qui consiste à susciter l'intérêt de l'interlocuteur en quelques minutes voire secondes -, en mettant en avant le casting, la thématique du film, une idée de partenariat avec une association locale dans le cadre de la politique d'animation du cinéma, etc. À l'inverse, certains exploitants programment "à l'aveugle», sur le simple nom d'un auteur, et n'ont alors pas besoin d'être convaincus.

Si la notion de différenciation est utile pour comprendre les stratégies d'acteurs, elle l'est d'autant plus dans la compréhension de l'identité des acteurs eux-mêmes et de leurs rapports de force. Les stratégies des programmateurs peuvent différer selon le film ou l'interlocuteur, mais nous observons la récurrence et la prédominance de l'adoption d'une posture spécifique constante caractéristique de leur personnalité. Par exemple, l'honnêteté. Le distributeur " honnête » cherche à acquérir la confiance de l'exploitant :

Il peut m'arriver de défendre le film du copain. Tout le monde ne le fait pas, mais parfois je le fais, je suis honnête. Si notre film n'était pas identifié parce qu'il est seulement passé à Locarno ${ }^{22}$, qu'il n'est pas dans les pré-visionnements régionaux, qu'il n'a pas encore obtenu le soutien de l'AFCAE ${ }^{23}$, et que donc on ne sait pas ce que c'est, personne n'en parle. Quand l'exploitant te dit qu'il prend celui dont tout le monde parle plutôt que le tien, et que toi tu l'as vu et qu'il t'a bouleversé, tu ne vas pas lui reprocher, tu lui dis que c'est normal et que tu comprends ${ }^{24}$.

15 La réputation d'honnêteté, qui se construit dans le temps, est importante pour installer de la confiance, car il s'agit du principal atout de certaines sociétés. D'autres peuvent faire, en quelque sorte, l'économie de ce travail relationnel parce qu'ils distribuent des films « forts » qui sont demandés par les exploitants, et fidélisent leurs auteurs.

Les programmateurs de salles et de films pensent leur programmation de manière unitaire ; les distributeurs prennent en compte les autres films qu'ils vont distribuer dans l'année, et les exploitants l'ensemble des films à l'affiche dans le cinéma ou la programmation mensuelle de la salle. Le distributeur peut ne pas avoir besoin de convaincre l'exploitant si le film est très demandé. En revanche, ce dernier peut accepter de programmer un film dont il peut supposer qu'il ne fera pas beaucoup d'entrées soit pour « rendre service » à un distributeur avec lequel il a l'habitude de travailler, ou bien dans une vision à plus long terme, accepter le film «faible » d'un distributeur, cette fois, pour obtenir son prochain film « fort » en jouant sur un potentiel conflit de loyauté en cas de refus.

17 Parler de loyauté n'est pas anodin ; les relations interpersonnelles sont très fortes dans le milieu, d'abord et surtout parce que c'est un petit milieu professionnel. Les sociétés de distribution ont un positionnement stratégique éditorial plus ou moins marqué, mais identifié par les exploitants. De ce fait, distributeurs et exploitants ont l'habitude de travailler régulièrement avec les mêmes personnes, parce qu'ils ont les mêmes goûts ou une ligne éditoriale proche - la frontière entre les deux restant souvent un peu floue -, ou qu'ils défendent une même vision du cinéma.

Les affinités entre types de salles et types de films se retrouvent au sein des pratiques dans le positionnement que les distributeurs et les exploitants adoptent les uns par rapport aux autres, faisant ressortir par là même le discours de ceux qui défendent la diversité versus les " usines à pop-corn" qui n'auraient pour objectif que le seul profit. 
Cette posture, qui dévoile la facette politique, voire militante, des acteurs de la diffusion qui se sentent investis d'une mission, se traduit également dans les négociations concernant la programmation. En effet, certains distributeurs sont habitués à travailler avec certaines salles, pour les raisons évoquées plus haut, parce qu'ils se doivent d'être solidaires en raison d'un engagement partagé, mais aussi parce qu'ils ont le sentiment de se comprendre, notamment du fait qu'ils peuvent connaître des difficultés économiques similaires. L'expression "la grande famille du cinéma ", généralement employée avec ironie, n'en est pas moins dénuée de sens lorsque l'on analyse les pratiques professionnelles et les discours portés sur celles-ci par les acteurs. C'est un milieu où tout le monde se tutoie :

Le vouvoiement est parfois méprisant, cela crée une distance, donne une impression de supériorité. Même quand tu n'as pas de feeling, tu tutoies. C'est en quelque sorte une posture de neutralité. Si on vouvoie, c'est qu'il y a un malaise $e^{25}$.

Les rencontres physiques ne sont pourtant pas si fréquentes, bien qu'il y ait des rendezvous annuels immanquables:

On se voit dans les festivals, dans les rendez-vous professionnels, donc forcément des liens se tissent. Il y a des histoires d'amour aussi! Il ne faut pas mettre trop d'affect, mais c'est difficile, moi je me suis fait beaucoup d'amis dans l'exploitation. À Cannes, lors des pré-visionnements, on voit les films ensemble donc on a leur ressenti, et après on mange ensemble, on boit des coups, donc on échange sur le film. Mes premiers rendez-vous consistaient à aller boire un café pour rencontrer les exploitants, se connaitre un peu, se raconter nos parcours professionnels et de vie, pourquoi la distribution, pourquoi l'exploitation ${ }^{26}$.

Si les rencontres physiques restent occasionnelles, le contact est hebdomadaire, voire pluri hebdomadaire, car ils gèrent également la circulation des copies. Ce contact très fréquent et constant instaure de fait une proximité entre les personnes, qui préfèrent se parler «en vrai » plutôt que par courriel, moyen de communication privilégié dans la quasi-totalité des relations interprofessionnelles d'autres secteurs: "Si tu n'aimes pas parler aux gens, faut pas faire ce métier. Parfois je fais un mail, et ils me rappellent, choqués, en me disant : "pourquoi tu m'as pas appelé ?!" ".

$\mathrm{Si}$, chez un grand nombre d'acteurs parmi les indépendants, la solidarité domine régulièrement, la concurrence pousse certains à utiliser des stratégies consistant à convaincre les exploitants de choisir leur film, car ceux du concurrent sont «mauvais»:

Moi, je ne fonctionne pas sur un rapport de force qui consiste à démonter le film de l'autre. Certains font ça, communément appelé « des techniques de gros ${ }^{27}$ ». Après quand tu apprends via les exploitants que tel distributeur a craché sur ton film pour pouvoir placer le sien, ce sont des pratiques qui se font, on est des humains... mais bon... la concurrence existe... C'est du commerce, il faut tirer son épingle $d u$ jeu, et parfois ça veut dire piquer une séance au copain ${ }^{28} .$.

21 Les tensions causées par la primauté d'enjeux d'ordre économique entre les acteurs engendrent régulièrement des conflits, principalement sur la question des séances nombre et horaire -, mais aussi sur la «tenue » des films - si l'exploitant s'engage à garder le film pendant trois semaines et qu'il décide finalement de le déprogrammer au bout de deux. Les accords passés entre distributeurs et exploitants peuvent ne pas toujours être respectés. En cas de refus de programmation pour les salles ou refus de ventes pour les distributeurs, la Médiatrice ${ }^{29}$ du cinéma peut être saisie. Par son rôle de conciliation, la Médiatrice du cinéma invite les deux parties en litige à trouver un accord amiable, pouvant aller jusqu'à l'injonction en cas de désaccord persistant ${ }^{30}$. Statistiquement, les deux tiers des saisines de la Médiatrice sont résolus par un accord 
amiable (en conciliation ou avant même la réunion). En effet, si la saisie de la Médiatrice est régulière - 96 saisines en moyenne par an ces dix dernières années -, il est important pour les distributeurs et les exploitants de soigner leur relation sur le long terme et de ne pas « s'embrouiller vraiment », parce que la programmation n'est pas l'histoire d'un seul film :

Quand tu as un film difficile à sortir, tu n'oublies pas les gens qui t'ont aidé toute l'année, et tu vois bien l'exploitant opportuniste qui va peut-être exposer mieux ton film sur une courte période, mais bon tu prends l'autre parce qu'il faut raisonner sur du long terme. C'est pour ça qu'il ne faut pas s'embrouiller ad vitam aeternam, parce que tu finis par avoir besoin de l'autre ${ }^{31}$.

\section{Conclusion : une relation durable}

Jeux stratégiques entre pouvoir économique et autorité symbolique, pour reprendre la formule de Michel de Certeau ${ }^{32}$, certains ne manquent pas de relever le caractère superficiel de cette relation qui reste avant tout une relation de travail :

Ce sont donc des discussions longues et douloureuses, mais qui paradoxalement se présentent souvent comme des rapports amicaux, finalement très superficiels. Quelque part, tout le monde se fait la bise, mais cela reste un commerce et chacun sert ses intérêts ${ }^{33}$.

Rapport de force ou solidarité, hostilité ou loyauté, les relations entre les distributeurs et les exploitants, parce qu'elles sont avant tout d'ordre commercial, combinent stratégies d'affrontement et stratégies de coopération. Dans l'acception de la théorie du conflit selon Georg Simmel, les rapports concurrentiels et le conflit sont créateurs de lien social, car ils ont un effet de socialisation. Cette forme de lutte, à penser donc comme une construction sociale, « oblige le concurrent, qui voit qu'il a un rival - et qui bien souvent n'entrerait pas en concurrence sans cela -, à aller au-devant et à se rapprocher de celui qu'on cherche à séduire, à se lier à lui, à étudier ses forces, ses faiblesses et ses caractéristiques, et à s'y adapter, à chercher toutes les passerelles qui pourraient relier sa propre personne et son propre travail au sien, ou à les établir ${ }^{34}$ ». En ce sens, le conflit favoriserait donc un équilibre social et la fabrication de l'organisation des rapports entre les acteurs.

Si le fonctionnement du secteur cinématographique peut sembler similaire à d'autres secteurs marchands - relations de pouvoir, importance du relationnel, conflits de loyauté, etc. -, sa spécificité peut résider dans le caractère mouvant des rapports de force à l'œuvre du fait de son économie prototypique. En outre, la tension entre art et industrie, spécifique au cinéma et plus largement aux industries culturelles, en soulève conséquemment une entre la nécessité de la rentabilité économique et la défense de la diversité culturelle. Les acteurs du secteur cinématographique ont tendance à induire que l'aspect économique prédomine toujours sur les relations parce qu'au fond, «c'est un marché ", mais l'analyse de leurs pratiques montre l'importance d'autres aspects d'ordre culturel et symbolique. En effet, défendre la diversité culturelle rime souvent avec le fait de programmer un film qui «ne marche pas », comparativement au nombre d'entrées que certains films dont l'objectif est uniquement économique peuvent atteindre. Cette spécificité permet d'expliquer par exemple le fait que certains ne cherchent pas à " écraser la concurrence ", mais comprennent et acceptent un refus de programmation de la part d'un exploitant par intérêt pour un autre film. 
En dépit donc de l'état de fait d'un marché très concurrentiel, la forte dimension culturelle du secteur cinématographique atténue les impératifs économiques des stratégies d'acteurs en les combinant avec d'autres logiques. On y retrouve à la fois des rapports interindividuels liés aux "conventions» du milieu artistico-culturel et une proximité des acteurs liée à la taille réduite du secteur qui tend à être structuré par les parcours et les caractères humains, et dans le même temps des enjeux cruciaux relatifs à la survie économique des acteurs sur un marché donné.

\section{BIBLIOGRAPHIE}

Olivier Bomsel, Gilles Le Blanc, La numérisation de l'industrie du cinéma, Rapport final, CERNA, mai 2002, $47 \mathrm{p}$.

Sarah Budex, «L'homme de l'ombre. Le programmateur, entre positionnement économique et représentations symboliques ", dans Claude Forest et Hélène Valmary (dir.) La vie des salles de cinéma, Paris, Presses Sorbonne-Nouvelle, Coll. Théorème, 2014, p.127-140.

Bruno Cailler, « D’une stratégie à l'autre, la démarche stratégique en question » dans Laurent Creton (dir.), Cinéma et stratégies : Économie des interdépendances, Paris, Presses Sorbonne-Nouvelle, Coll. Théorème, 2008, p. 149-178.

Florent Champy, La sociologie des professions, Paris, Presses Universitaires de France, 2009, 258 p.

Laurent Creton, Économie du cinéma : perspectives stratégiques, Armand Colin, Cahors, Armand Colin cinéma, 2005 (3ème édition), 287 p.

Michel Crozier, Erhard Friedberg, L'acteur et le système, Paris, Éditions du Seuil, 1977, 500 p.

Michel de Certeau, L'invention du quotidien, 1. Arts de faire, Paris, Gallimard, Coll. Folio/Essai, 1990, 349 p.

Claude Forest, « Mettre un film à l'affiche », dans Laurent Creton (dir.), Cinéma et stratégies : Économie des interdépendances, Paris, Presses Sorbonne-Nouvelle, Coll. Théorème, 2008, p. 15-32.

Jean-Michel Gévaudan, « Films et salles : des affinités électives ? », dans Laurent Creton (dir.), Cinéma et stratégies : Économie des interdépendances, Paris, Presses Sorbonne-Nouvelle, Coll. Théorème, 2008, p. 63-74.

Hélène Laurichesse, « Le marketing : les rapports balbutiants entre l'amont et l'aval », dans Isabelle Fauvel, Développer un projet pour le cinéma, Paris, Dixit, 2001, p. 137-154.

Bernard Miège, L'information-communication, objet de connaissance, De Boeck \& INA, Bruxelles, 2004,248 p.

Marc-Antoine Robert et Céline Merrien, « Dynamique et dynamisme du distributeur indépendant ", dans Laurent Creton (dir.), Cinéma et (in)dépendance : une économie politique, Paris, Presses Sorbonne-Nouvelle, Coll. Théorème, 1998, p. 61-81.

Georg Simmel, Le Conflit, Belval, Éditions Circé, coll. Poche, 2003 (1995), 158 p. 


\section{NOTES}

1. Le taux de location est toujours de 50/50 en première semaine, et diminue généralement de 10 en 10 au fur et à mesure des semaines en faveur de l'exploitant.

2. «La programmation de salles, lorsqu'elle n'est pas assurée directement par les entreprises propriétaires du fonds de commerce, est effectuée par un groupement ou une entente de programmation. À noter que certains groupements ou ententes programment les salles dont ils sont propriétaires. En 2017, les onze groupements et ententes nationaux agréés programment 3119 écrans, soit 52,7 \% de l'ensemble des écrans. » (cf. Bilan 2017, les dossiers du CNC n³38, mai 2018, p. 85).

3. Lorsque le distributeur se réfère à "la salle » ou à « l'exploitant » il faut comprendre par-là l'exploitant programmateur, même si le terme n'est pas mentionné. Le lieu de projection se substitue aux potentiels intermédiaires décisionnaires. Le programmateur devient « la salle », ou parfois une entité, telle « UGC » ou « Gaumont ».

4. Un contrat de location papier signé est encore établi pour les projections non commerciales, sans billetterie CNC.

5. Entendons par là bobine $35 \mathrm{~mm}$, car même s'il y a un DCP physique, celui-ci n'a plus besoin de rester dans le cinéma pour être projeté et peut donc circuler dans d'autres salles indépendamment des séances de projection.

6. Claude Forest, "Mettre un film à l'affiche ", dans Laurent Creton (dir.), Cinéma et stratégies : Economie des interdépendances, Paris, Presses Sorbonne-Nouvelle, Coll. Théorème, 2008, p. 15-32.

7. Sarah Budex, «L'homme de l'ombre. Le programmateur, entre positionnement économique et représentations symboliques ", dans Claude Forest et Hélène Valmary (dir.) La vie des salles de cinéma, Paris, Presses Sorbonne-Nouvelle, Coll. Théorème, 2014, p.127-140.

8. Cet article a été réalisé à partir du matériau récolté dans le cadre d'une thèse en cours intitulée Les acteurs de la diffusion cinématographique en salles en France au prisme du numérique : enjeux socio-économiques d'un secteur en mutation (2000-2016) dirigée par Laurent Creton. L'étude s'appuie une cinquantaine d'entretiens semi-directifs réalisés entre 2011 et 2018 auprès d'une cinquantaine de professionnels du secteur de la diffusion, d'une analyse détaillée de la presse professionnelle entre 2000 et 2018 et de rencontres régulières avec distributeurs et exploitants au sein d'un groupe de travail sur la programmation.

9. Florent Champy, La sociologie des professions, Paris, Presses Universitaires de France, 2009, 258 p.

10. Michel Gomez, ibid., p. 7.

11. David Vandiedonck, «Bernard Miège: L'information-communication, objet de connaissance ", Études de communication, 28 | 2005, p. 185-188.

12. Olivier Bomsel, Gilles Le Blanc, La numérisation de l'industrie du cinéma, Rapport final, CERNA, mai 2002, p. 41.

13. Le potentiel commercial reste relatif au film et au type de distributeur. Ainsi, un film visant le million d'entrées et un autre la barre des 100000 peuvent tous deux être considérés comme « forts », dépendant de l'horizon d'attente que se fixent les distributeurs.

14. Marc-Antoine Robert et Céline Merrien, « Dynamique et dynamisme du distributeur indépendant ", dans Laurent Creton (dir.), Cinéma et (in)dépendance : une économie politique, Paris, Presses Sorbonne-Nouvelle, Coll. Théorème, 1998, p. 71. 
15. Gaspard va au Mariage, réalisé par Anthony Cordier et distribué par Pyramide Distribution.

16. Wonder Wheel, réalisé par Woody Allen et distribué par Mars Films.

17. Phantom Thread, réalisé par Paul Thomas Anderson et distribué par Universal Picture International France.

18. l'Apparition, réalisé par Xavier Giannoli et distribué par Memento Films Distribution.

19. Michel Crozier, Erhard Friedberg, L'acteur et le système, Paris, Editions du Seuil, 1977, p. 23.

20. Michel Crozier, Erhard Friedberg, ibid., p. 90.

21. Hélène Laurichesse, "Le marketing : les rapports balbutiants entre l'amont et l'aval », dans Isabelle Fauvel, Développer un projet pour le cinéma, Paris, Dixit, 2001, p 137-154.

22. Le festival international du Film de Locarno met en avant le cinéma dit « d'auteur ». Il est important pour la profession mais peu connu du grand public et a fortiori ne donne pas de grande visibilité au film ou ne peut que difficilement être utilisé comme argument promotionnel auprès du public.

23. Association Française des Cinémas d'Art et Essai.

24. Entretien avec une programmatrice d'une société de distribution indépendante, janvier 2018.

25. Entretien, ibid.

26. Entretien, ibid.

27. Les circuits d'exploitation exercent régulièrement des pressions sur les distributeurs pour obtenir une exclusivité sur une même zone de chalandise par exemple.

28. Entretien, ibid.

29. Institué en 1983 et nommé pour un mandat de quatre ans renouvelable, le Médiateur du cinéma est depuis 2015 la conseillère d'État et Présidente du CA de l'Institut Nationale de l'Histoire de l'Art Laurence Franseschini.

30. Cf. Rapport annuel d'activité du Médiateur du Cinéma 2017, disponible sur: http:// www.lemediateurducinema.fr/Mediateur/dernier-rapport.htm

31. Entretien, ibid.

32. Michel de Certeau, L'invention du quotidien, 1. Arts de faire, Paris, Gallimard, Coll. Folio/Essai, 1990, 349 p.

33. Entretien avec un programmateur et distributeur d'une entente de programmation, janvier 2013.

34. Georg Simmel, Le Conflit, Belval, Editions Circé, coll. Poche, 2003 (1995), p.76.

\section{RÉSUMÉS}

Cet article se propose d'étudier la relation entre distributeurs de films et exploitants de salles de cinéma par l'examen de leurs pratiques professionnelles et l'analyse des discours sur leurs pratiques. Il s'agit de montrer que leur relation ne relève pas seulement d'un enjeu commercial le distributeur loue une copie du film que l'exploitant a choisi de programmer - mais qu'il s'agit d'une relation de pouvoir qui se construit autour de rapports de force différents selon les types d'acteurs mis en relation et les films à programmer, combinant à la fois stratégies d'affrontement et stratégies de coopération.

Based on a series of interviews, this article focuses on the relationship between film ditributors and exhibitors through the study of their professional practices. Their exchanges go beyond simple questions of commerce, where the distributor rents a copy of the film to the exhibitor, 
who then schedules screenings. Combining strategies of confrontation and cooperation, these relationships also depend on the relative power of both parties, as well as the films being programmed.

\section{AUTEUR}

\section{CLÉMENCE ALLAMAND}

Clémence Allamand est doctorante et ATER à l'université Sorbonne Nouvelle - Paris 3. Elle prépare une thèse sur les enjeux socio-économiques des acteurs de la diffusion cinématographique en France au prisme du numérique sous la direction de Laurent Creton (EA 185 IRCAV / ED 267). 